\title{
Road Detection Using Classification Algorithms
}

\author{
Şafak Altay Açar ${ }^{1 *}$, Şafak Bayır ${ }^{2}$ \\ 1 The Department of Electronics and Computer Education, Faculty of Technical Education, Karabük \\ University, Karabük, Turkey. \\ ${ }^{2}$ Department of Computer Engineering, Faculty of Engineering, Karabük University, Karabük, Turkey. \\ * Corresponding author. Tel.: 05546174183; email: safakaltay@karabuk.edu.tr \\ Manuscript submitted January 9, 2015; accepted March 5, 2015. \\ doi: $10.17706 /$ jcp.10.3.147-154
}

\begin{abstract}
In this study, we present a road detection method. Proposed method consists of two phases. In the first phase, a binary image is obtained by utilizing greyscale transformation and thresholding processes. In the second phase, K-Nearest Neighbours and Naive Bayes classifiers are applied on image by utilizing colour features. Road and non-road regions are determined and these two classifiers are compared with each other. Google Maps satellite images are used since they are easily accessible on the Internet. C\# based software is developed for realizing and testing the methods mentioned above.
\end{abstract}

Key words: K-nearest neighbours, naive bayes, road detection.

\section{Introduction}

Since the developments in satellite technology increases rapidly, various academic studies on satellite images have been carried out widely nowadays. Satellite images are used in many areas such as transportation, meteorology, geology, agriculture, regional planning etc. Road detection is one of the most important topics among them; therefore, obtaining information related to roads is a strategically important issue in urban areas. Public institutions, organizations and corporations such as hospitals, fire departments, police stations, schools etc. are connected each other by roads; consequently, analysing the existence of the roads on satellite images becomes gradually more important.

When the relevant literature is taken into consideration, it is seen that many academic studies are made on road detection and extraction by utilizing satellite images. Xu et al. [1] propose an approach to extract roads using watershed dual-thresholds algorithm and multi-weighted method. They state that proposed algorithm has a high precision and a good robustness in the various types of urban road extraction. Poullis and You [2] propose an algorithm for road networks detection and extraction. They combine perceptual grouping theory (Gabor filtering, tensor voting) and optimized segmentation techniques (global optimization using graph-cuts). Proposed system achieves a minimum success rate of an average of $69.3 \%$. A new method is presented by Movaghati et al. [3] which merges extended Kalman filter and particle filter for road extraction. They use two different satellite images in their experiments. The correctness values of both images are 0.98 and the completeness values are 0.92 and 0.85 respectively. Yuan et al. [4] propose a road detection algorithm based on locally excitatory globally inhibitory oscillator networks. Completeness is computed as $0.83,0.89$ and 0.59 for three satellite images and correctness values are $0.71,0.62$ and 0.65 respectively. Moreover, they indicate that a comparison with other methods shows that proposed method produces very competitive extraction results. Another new method is presented by Arafat et al. [5] which 
detects roads using multiple simple colour space components. They state that proposed system gives good and accurate results on the main road segments. Ünsalan and Sirmacek [6] present a study to detect road networks. System consists of three phases which are probabilistic road centre detection, road shape extraction, and graph-theory-based road network formation. After graph refinement process, they calculate completeness, correctness and quality for all test images as $0.75,0.74$ and 0.59 respectively. They reveal that obtained results indicate that their system can be used in detecting the road network on relevant images in a reliable and fast manner. Peeters and Etzion [7] propose an approach based on geographic information system for urban objects recognition. They use context-based recognition for a case study and they find out the overall classification accuracy as $80.30 \%$. A study is presented by Revathi and Sharmila [8] which extracts roads using level set and mean shift methods. They compare these methods and mean shift performs (completeness value: 98.28\% and correctness value: 95.31\%) better than level set method (completeness value: 93.9\% and correctness value: 87.89\%). Abraham and Sasikumar [9] present an approach including a wavelet based segmentation method combined with a fuzzy based intelligent system for road network extraction. According to them, acceptable accuracy for the extracted features is obtained even for degraded and poor quality images. They obtained the quality percentages of the reference images as follows: image 1 (degraded) as 78.3\%, image 2 (noise unaffected) as $87.7 \%$, image 3 (noise affected) as $84.2 \%$, image 4 (low resolution) as $65.4 \%$, image 5 (without blurring) as $92.1 \%$ and image 6 (with blurring) as $90.8 \%$. Gürbüz and Alatan [10] present a study based on tubularity tracking and graph cuts for road detection. They say that the most important feature of the proposed method is its local peak detection filter and the obtained average accuracy is $74.30 \%$.

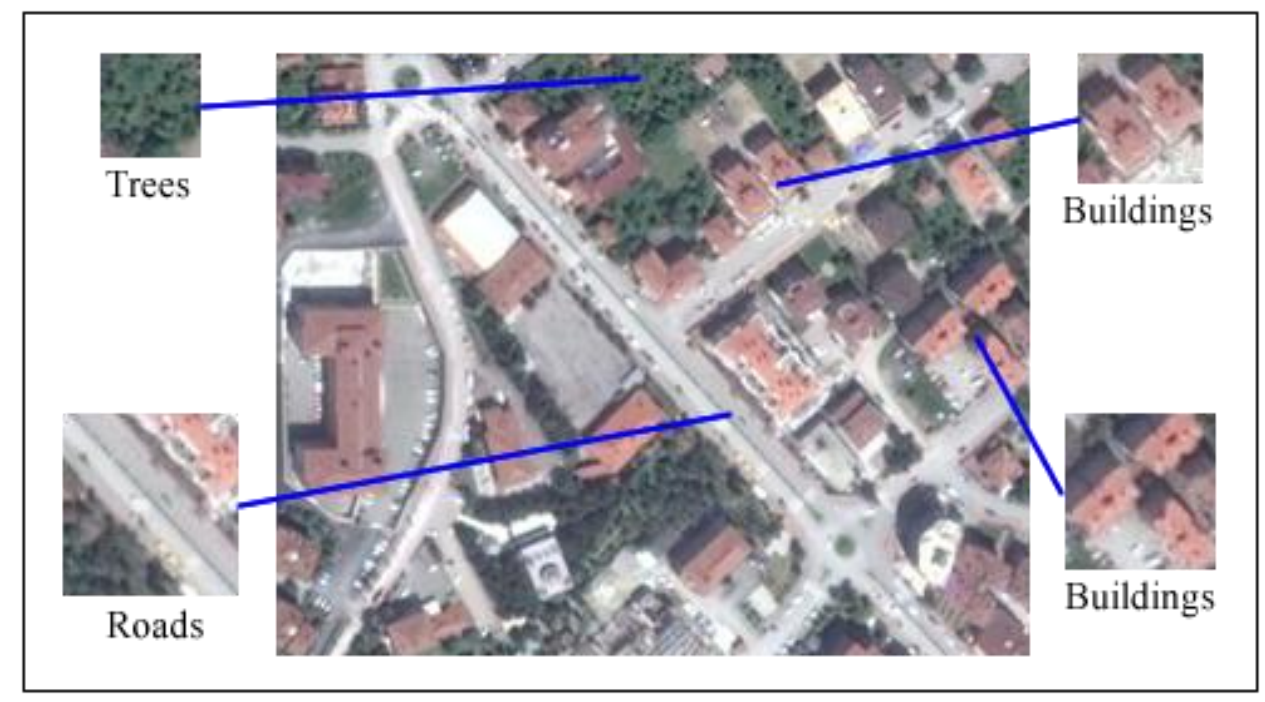

Fig. 1. Road and non-road regions.

Furthermore, there are few studies which use classifiers. Inglada [11] presents a study based on support vector machines for recognition of man-made objects such as highways and railways and emphasizes that the results show the possibility of discrimination of several classes of objects with classification rates higher than $80 \%$. Mokhtarzade and Zoej [12] propose an approach to detect roads using artificial neural networks. Overall accuracy values are approximately higher than 90\%. Das et al. [13] present an approach to extract roads using probabilistic support vector machines, salient features which are distinct spectral contrast and locally linear trajectory, segment linking and non-road regions eliminating. Final output of the proposed method's average completeness and correctness values are $89 \%$ and $93 \%$ respectively. Sirmaçek and Ünsalan [14] present a road detection approach. They use colour features and one-class classification. They calculate true positive detection performance rate for two different data sets. These are $81.64 \%$ and 
66.33\%. Kirthika and Mookambiga [15] propose an approach to extract road network using artificial neural network and the road detection correctness coefficient is calculated as 80.71. Shi et al. [16] propose an approach to extract urban main road centreline. Support vector machines, general adaptive neighbourhood, local Geary's C algorithms and local linear kernel smoothing regression are utilized. Method is compared with other four existing road extraction methods and it is said that the proposed method is more suitable for urban main-road centreline extraction.

This paper presents a road detection method based on classification algorithms. Proposed method consists of two phases. In the first phase, a binary image is obtained by utilizing greyscale transformation and thresholding processes. In the second phase, K-Nearest Neighbours and Naive Bayes classifiers are applied on image by utilizing colour features. As a result of these processes, road and non-road regions are determined.

Road and non-road regions such as buildings, trees etc. are shown in Fig. 1.

\section{Greyscale Transformation and Thresholding}

When we analyse satellite images, we see that roads are brighter than other regions and after thresholding process, roads turn into white colour. In order to use this feature, we apply thresholding to images after greyscale transformation and classification process is applied only to white colour regions in order to reduce runtime.

We use Otsu thresholding method [17]. In this method, binary image is obtained using threshold value (t1). Pixels which have a value less than $\mathrm{t} 1$ are accepted as black and other pixels are accepted as white.

During thresholding process, red, green and blue colour values of image pixels are analysed. Differences between these colours' values are calculated separately. If one of them is higher than threshold value (t2), related pixel becomes black regardless of $\mathrm{t} 1$. After experimental studies, $\mathrm{t} 2$ is determined as 13 . Also, if a pixel's all colour values is higher than threshold value (t3), related pixel becomes black regardless of $t 1$. After experimental studies, $\mathrm{t} 3$ is determined as 245. By means of these two threshold values ( $\mathrm{t} 2$ and $\mathrm{t} 3$ ), regions which have a low probability of being road are eliminated.

\section{Road Detection}

A town's satellite images are obtained from Google Maps. Little square pieces are extracted from two of them. Some of these pieces include road regions and others include non-road regions. Images which are not utilized for extracting pieces are used for road detection. Total number of pieces is 120 (60 roads, 60 non-roads) and size of a one piece is $15 \times 15$ pixels. Sample pieces are shown in Fig. 2 .

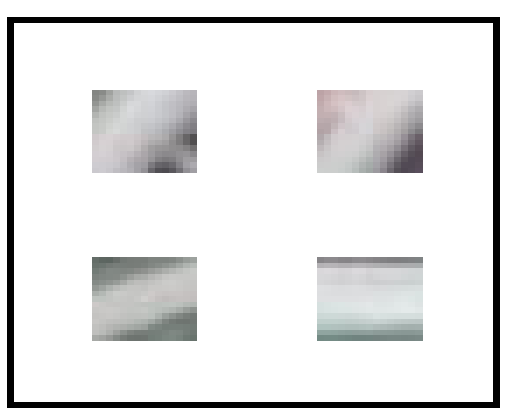

(a)

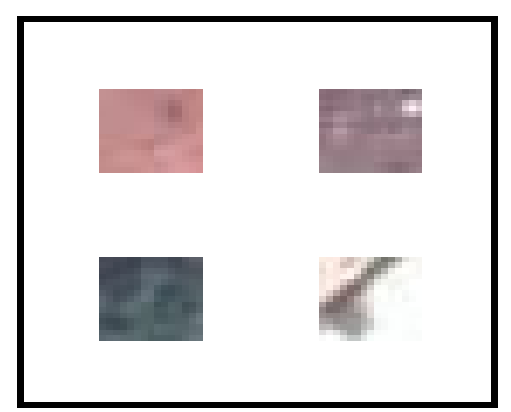

(b)

Fig. 2. (a) Road pieces (b) non-road pieces.

Classification process is applied by utilizing colour features of pieces. We divide piece's pixel colour values into three ranges. These are $[0,85],[86,170]$ and $[171,255]$. Mean values are calculated one by one 
for three ranges and red-green-blue colour values. As a result, we obtain nine different mean values from one piece. Used formulas to obtain mean values are defined as follows:

$$
\begin{array}{ll}
\mu_{C 1}=\sum_{1}^{N_{C 1}} C 1_{(x, y)} / N_{C 1} & C 1_{(x, y)} \in[0,85] \\
\mu_{C 2}=\sum_{1}^{N_{C 2}} C 2_{(x, y)} / N_{C 2} & C 2_{(x, y)} \in[86,170] \\
\mu_{C 3}=\sum_{1}^{N_{C 3} C 3_{(x, y)} / N_{C 3}} & C 3_{(x, y)} \in[171,255]
\end{array}
$$

$C 1_{(x, y)}, C 2_{(x, y)}$ and $C 3_{(x, y)}$ denote piece's total pixel colour values but each one relates different value range. $N_{C 1}, N_{C 2}$ and $N_{C 3}$ denote number of values. Equations (1), (2) and (3) are calculated for red-green-blue colour values as explained above. Calculated mean values are used for classification.

We use two different classifiers for road detection. One of them is K-Nearest Neighbours and other one is Naive Bayes.

\subsection{K-Nearest Neighbours Classifier}

K-Nearest Neighbours algorithm is performed by shifting a $N \times N$ pixels block on satellite image. Shifting process starts on $(0,0)$ point and shifting distance is 5 pixels. Block moves 5 pixels every step as shown in Fig. 3 and blue squares denote moving block.

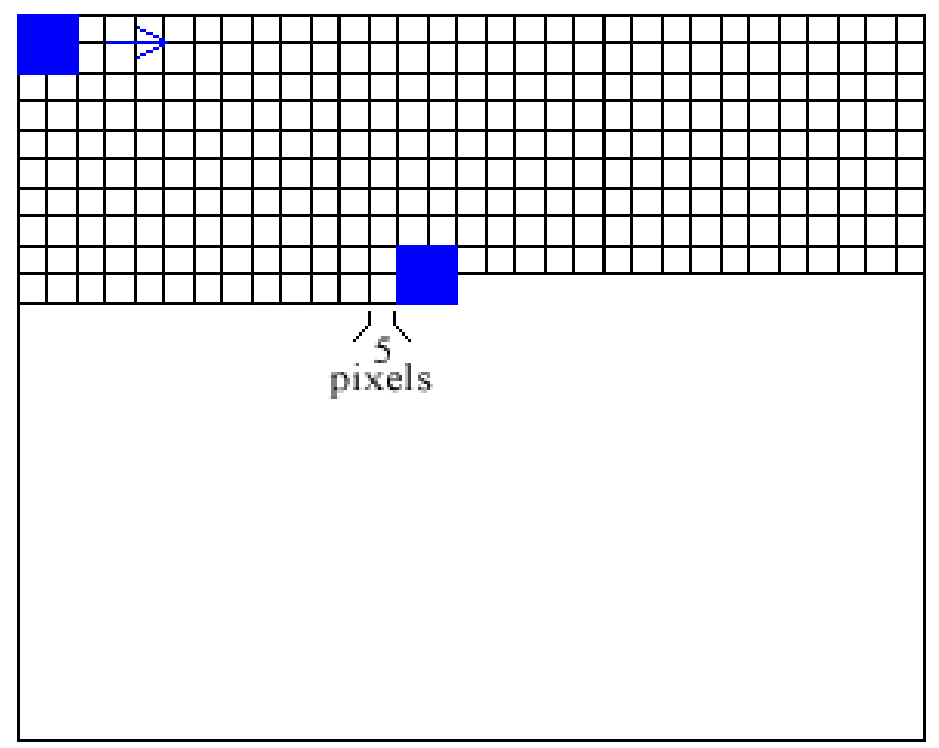

Fig. 3. Moving block.

In every step, binary image is checked firstly. If binary image's region which corresponds to block has at least $40 \%$ white colours, classification is applied on block's region. There are two classes as road and non-road. We use Euclidean distance for class selection. Block's nine mean values are calculated by utilizing (1), (2) and (3) and the closest 7 pieces are determined. If at least 6 of them are roads, block's region is classified as road.

Algorithm is carried out and calculated separately for two different block sizes. These are $15 \times 15$ and $10 \times 10$ pixels. $10 \times 10$ pixels are extracted from centre of these pieces $(15 \times 15$ pixels $)$.

\subsection{Naive Bayes Classifier}

Naive Bayes algorithm is performed in the same way as K-Nearest Neighbours. There is only one difference in class selection phase. We divide nine mean values into three ranges as follows: 


$$
\begin{array}{r}
f 1\left(\mu_{C 1}\right)=\left\{\begin{array}{lr}
1, & \mu_{C 1}<29 \\
2, \mu_{C 1} \geq 29 \text { and } \mu_{C 1}<58 & \\
3, \mu_{C 1} \geq 58 \text { and } \mu_{C 1} \leq 85
\end{array}\right. \\
f 2\left(\mu_{C 2}\right)=\left\{\begin{array}{lr}
1, & \mu_{C 2}<115 \\
2, & \mu_{C 2} \geq 115 \text { and } \mu_{C 2}<143 \\
3, \mu_{C 2} \geq 143 \text { and } \mu_{C 2} \leq 170
\end{array}\right. \\
f 3\left(\mu_{C 3}\right)=\left\{\begin{array}{lr}
1, & \mu_{C 3}<200 \\
2, & \mu_{C 3} \geq 200 \text { and } \mu_{C 3}<228 \\
3, & \mu_{C 3} \geq 228 \text { and } \mu_{C 3} \leq 255
\end{array}\right.
\end{array}
$$

Probabilities are calculated by utilizing these values and the block's region is classified afterwards.

\section{Experimental Results}

We evaluate proposed method on satellite images. 10 images of $390 \times 300$ pixels are used. K-Nearest Neighbours and Naive Bayes classifiers are compared with each other. Two different block sizes $(15 \times 15$ and $10 \times 10$ pixels) are utilized. A sample of results is shown in Fig. 4 .

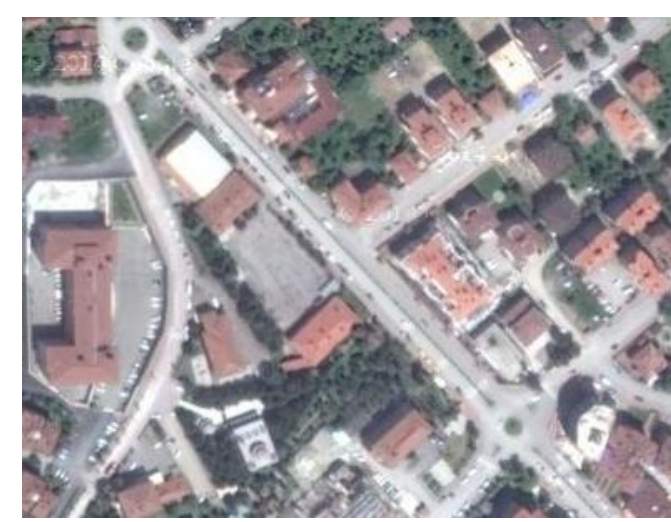

(a)

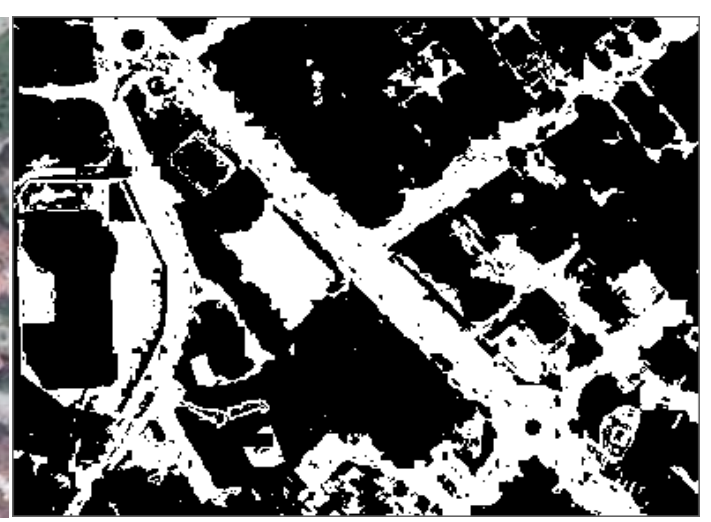

(b)

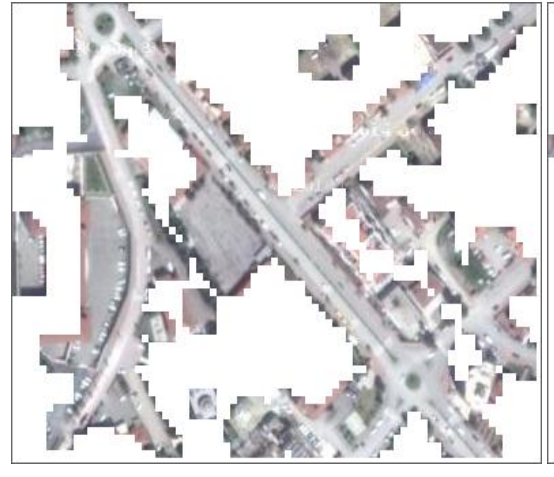

(c)

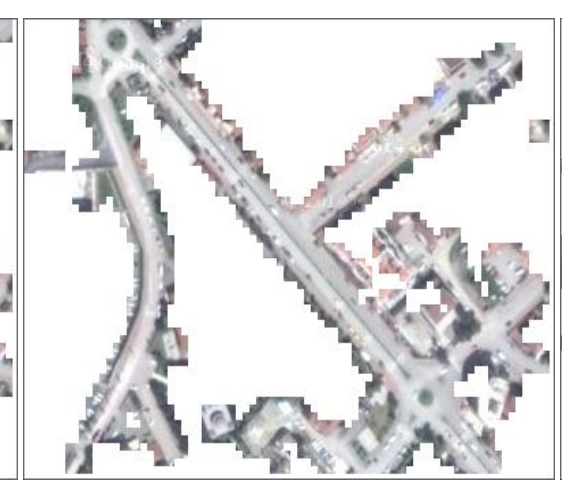

(d)

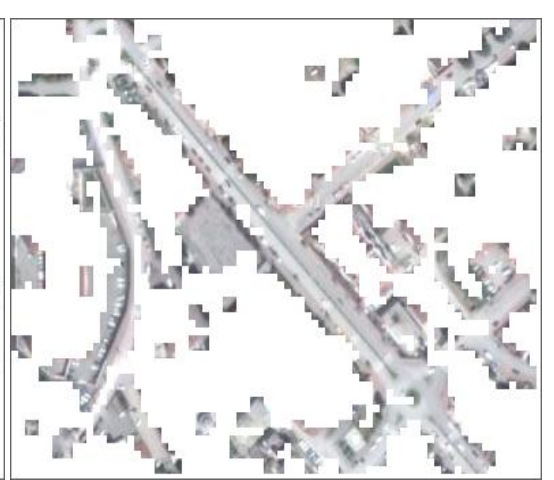

(e)

Fig. 4. (a) Original image, (b) binary image, (c) result of k-nearest neighbours (block size:15×15 pixels), (d) result of naive bayes (block size:15×15 pixels), (e) result of k-nearest neighbours (block size:10×10 pixels).

Tables 1, 2, 3 and 4 report evaluations of classifiers using completeness and correctness measures [18]. In this study, "completeness" represents the ratio of the pixel's number of the correctly extracted roads to the pixel's number of the reference roads and "correctness" represents the ratio of the pixel's number of correctly extracted roads to the pixel's number of all extracted roads. We use Google Maps to determine reference roads. Besides, we multiply results by 100 to obtain percent values. Results of Naive Bayes which 
are obtained using $10 \times 10$ block size are not satisfying; therefore, the results are not reported within the settings of this study.

Table 1. Evaluation of K-Nearest Neighbours Classifier (Block Size: $15 \times 15$ Pixels)

\begin{tabular}{ccc}
\hline Image & $\begin{array}{c}\text { Completeness } \\
(\%)\end{array}$ & $\begin{array}{c}\text { Correctness } \\
(\%)\end{array}$ \\
\hline 1 & 88.11 & 26.39 \\
2 & 76.67 & 27.95 \\
3 & 77.73 & 43.01 \\
4 & 84.75 & 34.07 \\
5 & 92.15 & 33.61 \\
6 & 84.18 & 32.91 \\
7 & 81.43 & 38.22 \\
8 & 78.92 & 36.47 \\
9 & 87.27 & 30.77 \\
10 & 77.58 & 27.44 \\
\hline
\end{tabular}

Table 2. Evaluation of Naive Bayes Classifier (Block Size: $15 \times 15$ Pixels)

\begin{tabular}{ccc}
\hline Image & $\begin{array}{c}\text { Completeness } \\
(\%)\end{array}$ & $\begin{array}{c}\text { Correctness } \\
(\%)\end{array}$ \\
\hline 1 & 80.03 & 31.41 \\
2 & 60.28 & 26.93 \\
3 & 57.59 & 46.84 \\
4 & 62.28 & 34.65 \\
5 & 49.36 & 42.79 \\
6 & 41.57 & 34.79 \\
7 & 65.31 & 40.14 \\
8 & 70.01 & 39.28 \\
9 & 68.68 & 32.19 \\
10 & 44.31 & 31.37 \\
\hline
\end{tabular}

Table 3. Evaluation of K-Nearest Neighbours Classifier (Block Size: $10 \times 10$ Pixels)

\begin{tabular}{ccc}
\hline Image & Completeness (\%) & Correctness $(\%)$ \\
\hline 1 & 72.02 & 31.35 \\
2 & 54.08 & 30.52 \\
3 & 71.43 & 51.65 \\
4 & 79.95 & 39.35 \\
5 & 82.27 & 43.05 \\
6 & 75.63 & 41.08 \\
7 & 68.23 & 45.42 \\
8 & 71.58 & 45.42 \\
9 & 66.49 & 33.48 \\
10 & 67.35 & 34.41 \\
\hline
\end{tabular}

Table 4 shows the calculated averages of completeness and correctness values. Average completeness values of K-Nearest Neighbours (block size: $15 \times 15$ pixels), Naive Bayes (block size: $15 \times 15$ pixels) and K-Nearest Neighbours (block size: $10 \times 10$ pixels) are $82.87 \%, 59.94 \%$ and $70.90 \%$ respectively. Average correctness values of K-Nearest Neighbours (block size: $15 \times 15$ pixels), Naive Bayes (block size: $15 \times 15$ pixels) and K-Nearest Neighbours (block size: $10 \times 10$ pixels) are as follows 33.08\%, 36.03\% and 39.57\%.

Table 4. Evaluation of Classifiers

\begin{tabular}{ccc}
\hline Classifier & Average Completeness (\%) & Average Correctness (\%) \\
\hline K-Nearest Neighbours (Block Size:15×15 pixels) & 82.87 & 33.08 \\
Naive Bayes (Block Size:15×15 pixels) & 59.94 & 36.03 \\
K-Nearest Neighbours (Block Size:10×10 pixels) & 70.90 & 39.57 \\
\hline
\end{tabular}

\section{Conclusion}

Road detection is achieved by using K-Nearest Neighbours and Naive Bayes classifiers. These two classifiers and two different block sizes are compared with each other. If we use $15 \times 15$ pixel block size, K-Nearest Neighbours algorithm has well completeness than Naive Bayes algorithm but it has less ratio of correctness. When we reduce block size, correctness increases but completeness decreases for K-Nearest 
Neighbours algorithm.

In future we will experiment with more features to improve our method in terms of correctness. Also we will use more classifiers to obtain more qualified evaluation.

\section{References}

[1] Xu, G., Zhang, D., \& Liu, X. (2009). Road extraction in high resolution images from Google Earth. Proceedings of International Conference on Information, Communications and Signal Processing (pp. 1-5).

[2] Poullis, C., \& You, S. (2010). Delineation and geometric modeling of road networks. ISPRS Journal of Photogrammetry and Remote Sensing, 65(2), 165-181.

[3] Movaghati, S., Moghaddamjoo, A., \& Tavakoli, A. (2010). Road extraction from satellite images using particle filtering and extended Kalman filtering. IEEE Transactions on Geoscience and Remote Sensing, 48(7), 2807-2817.

[4] Yuan, J., Wang, D., Wu, B., Yan, L., \& Li, R. (2011). LEGION-based automatic road extraction from satellite imagery. IEEE Transactions on Geoscience and Remote Sensing, 49(11), 4528-4538.

[5] Arafat, S. Y., Butt, A. Y., \& Liagat, N. (2011). Automatic road detection using MCSC. International Multitopic Conference (pp. 126-131).

[6] Ünsalan, C., \& Sirmacek, B. (2012). Road network detection using probabilistic and graph theoretical methods. IEEE Transactions on Geoscience and Remote Sensing, 50(11), 4441-4453.

[7] Peeters, A., \& Etzion, Y. (2010). Automated recognition of urban objects for morphological urban analysis. Computers, Environment and Urban Systems, 36(6), 573-582.

[8] Revathi, M., \& Sharmila, M. (2013). Automatic road extraction using high resolution satellite images based on level set and mean shift methods. International Conference on Computing, Communications and Networking Technologies (pp. 1-7).

[9] Abraham, L., \& Sasikumar, M. (2013). A fuzzy based road network extraction from degraded satellite images. Proceedings of International Conference on Advances in Computing, Communications and Informatics (pp. 2032-2036).

[10] Gürbüz, Y. Z., \& Alatan, A. A. (2014). Uydu görüntülerinde tüpsellik takibi üzerinden otomatik yol tanıma. Proceedings of Signal Processing and Communications Applications Conference (pp. 2190-2193).

[11] Inglada, J. (2007). Automatic recognition of man-made objects in high resolution optical remote sensing images by SVM classification of geometric image features. Photogrammetry and Remote Sensing, 62(3), 236-248.

[12] Mokhtarzade, M., \& Valadan Zoej, M. J. (2007). Road detection from high-resolution satellite images using artificial neural networks. Applied Earth Observation and Geoinformation. 9(1), 32-40.

[13] Das, S., Mirnalinee, T. T., \& Varghese, K. (2011). Use of salient features for the design of a multistage framework to extract roads from high-resolution multispectral satellite images. IEEE Transactions on Geoscience and Remote Sensing, 49(10), 3906-3931.

[14] Sırmaçek, B., \& Ünsalan, C. (2011). Road detection from remotely sensed images using color features. Proceedings of International Conference on Recent Advances in Space Technologies (pp. 112-115).

[15] Kirthika, A., \& Mookambiga, A. (2011). Automated road network extraction using artificial neural network. Proceedings of International Conference on Recent Trends in Information Technology (pp. 1061-1065).

[16] Shi, W., Miao, Z., \& Debayle, J. (2014). An integrated method for urban main-road centerline extraction from optical remotely sensed imagery. IEEE Transactions on Geoscience and Remote Sensing, 52(6), 3359-3372. 
[17] Otsu, N. (1979). A thresholding selection method from gray-level histograms. IEEE Transactions on Systems, Man and Cybernetics, 9(1), 62-66.

[18] Laptev, I., Mayer, H., Lindeberg, T., Eckstein, W., Steger, C., \& Baumgartner, A. (2000). Automatic extraction of roads from aerial images based on scale space and snakes. Machine Vision and Applications, 12, 23-31.

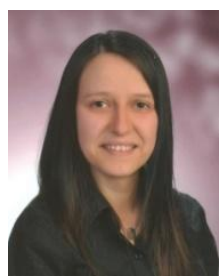

Şafak Altay Açar was born in Erzurum, Turkey on May 14, 1986. She is a PhD student at Karabük University, in the Computer Engineering Department. Also she is currently a research assistant at Karabük University, in the Electronics and Computer Education Department. Her research interest includes image processing, pattern recognition and artificial intelligence.

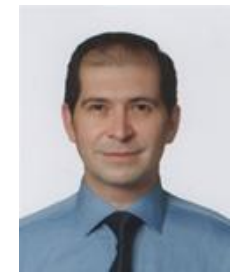

Şafak Bayır was born in Berlin, Germany on March 21, 1973. He is an assistant professor doctor in the Computer Engineering Department at Karabük University. His research interest includes educational technology and instructional technology. 